\title{
Armstrong on Quantities and Resemblance
}

\author{
Philosophical Studies (2007) 136: 385-404
}

\begin{abstract}
Resemblances obtain not only between objects but between properties. Resemblances of the latter sort - in particular, resemblances between quantitative properties - prove to be the downfall of David Armstrong's well-known theory of universals. This paper examines Armstrong's efforts to account for such resemblances, and explores several ways one might extend the theory in order to account for quantity. I argue that none succeed.
\end{abstract}

A theory of universals takes at face value the idea that things share properties. Such a theory holds that universals can be instantiated by numerically distinct objects. One of the natural applications of this theory is to explain how two things resemble one another, and thus to offer an answer to the so-called Problem of Resemblance: two things intrinsically resemble one another if and only if they share some of their universals. ${ }^{1}$

David Armstrong claims that universals provide the only tenable account of resemblance, because they provide the only reductive account (see Armstrong). ${ }^{2}$ But whether universals provide an attractive analysis of resemblance hinges on a crucial question: can a theory of universals account for resemblance relations among properties as well as resemblance relations among objects? Armstrong believes so. He offers an account according to which the more parts two properties share, the more similar they are. ${ }^{3}$

This strategy is fatally flawed. As a result, I argue, a theory of universals cannot count an analysis of resemblance among its virtues. Since one of its alleged strengths is an elegant and reductive analysis of resemblance, the failure to produce such an account is a mark against the theory. (I will not be weighing other costs and benefits here.)

In this paper I will look at how Armstrong's theory deals with quantitative properties, particularly those of classical mechanics. I do this for three reasons. First, Armstrong himself claims that universals are in a unique position to accommodate quantitative properties. ${ }^{4}$ Second, a world where the laws of classical mechanics hold is metaphysically possible, and Armstrong should be able to

\footnotetext{
${ }^{1}$ In this paper I am interested only in intrinsic resemblance, not extrinsic resemblance. For instance, I do not address cases where there is some sense in which two things resemble (perhaps each has the property of being five feet from a poodle), but where this resemblance does not arise from the intrinsic properties of each object alone.

${ }^{2}$ See Armstrong (1978) and (1989a).

${ }^{3}$ See Armstrong (1988) and (1989a, pg. 101-105).

${ }^{4}$ See Armstrong (1989a, pg. 101).
} 
account for such a world. Third, if Armstrong's theory cannot accommodate the properties of classical mechanics, there is little hope it will be able to accommodate the quantitative properties of more sophisticated physical theories.

\section{Armstrong's Picture: Resemblance as Partial Identity}

On Armstrong's picture, universals are sparse; they carve nature at the joints. The paradigmatic universals are the fundamental quantities expressed by predicates in an ideal physics. ${ }^{5}$ Gruesome predicates have no correlates in the world of universals. Armstrong is especially conservative with his ontology of higher-order universals, or universals instantiated by universals. (The relation of nomic necessitation is one of the few higher-order relations he allows.) Although positing higher-order universals may seem a natural way to account for property resemblance, Armstrong has several reasons to reject this approach. ${ }^{6}$ As a result, he instead proposes a different strategy to account for resemblances among properties.

Armstrong claims that universals can be constituents of other universals, just as objects can be parts of other objects. Universals made up of constituents are structural universals, while universals with no constituents are simple universals. On his account, the structure of universals mirrors the structure of the objects that instantiate them. Any object that instantiates a structural universal must have proper parts which instantiate that universal's constituents. Consider a structural universal $F$ with constituents $F_{1}$ through $F_{n}$. If object $a$ instantiates $F$, then $a$ must have numerically distinct proper parts $a_{1}$ through $a_{n}$ which instantiate $F_{1}$ through $F_{n}$, respectively. ${ }^{7}$

Two structural universals resemble one another to the extent to which they share constituents. If two universals do not share any constituents, they do not resemble one another in any respect. If they share at least one constituent, they

\footnotetext{
${ }^{5}$ See Armstrong (1988, pg. 87).

${ }^{6}$ One reason is the desire for ontological parsimony. Another is the fact that the natural candidates for such higher-order universals are instantiated necessarily by first-order universals, which is at odds with Armstrong's combinatorial view of possibility. See Armstrong (1978, pg. 105-108), (1983), and (1989b) for more discussion.

${ }^{7}$ If one axiomatizes the part-whole relation using a mereology that abandons the axiom of unique fusion, then Armstrong's constituency relation can be identified with the mereological relation of part to whole. On the other hand, if the part-whole relation is constitutively tied to the unique fusion axiom of classical mereology, then Armstrong must accept the constituency relation as a primitive in his ontology, albeit one that closely approximates the notion of traditional parthood. See Lewis (1986) for discussion.
} 
resemble one another at least somewhat. The more constituents they share, the more similar they are: all resemblance is reduced to partial or whole identity.

Armstrong claims that every quantitative property is a structural universal. Think of quantitative universals as Russian nesting dolls. Within each doll there is a smaller doll, and a smaller one, ad infinitum. The largest doll "contains" all the other dolls: it shares many nested dolls with the second-largest doll, slightly fewer nested dolls with the third-largest doll, and so on. In terms of the quantity of shared dolls, the largest is more similar to the second-largest than to the thirdlargest. Likewise for quan titative universals - every quantitative property has an infinite number of "nested" constituent universals. Intuitively, the more constituents two quantitative universals share, the more similar they are. For example, the five grams mass universal shares many constituents with the four grams mass universal; hence the property of five grams mass closely resembles the property of four grams mass.

Armstrong's remarks can be usefully formalized by the following two principles. The first principle provides an intuitively plausible way to determine the constituents of a structural universal. Call it the constituency principle: a universal $x$ is a constituent of universal $y$ iff every object in every possible world that instantiates $y$ has some proper part that instantiates $x$. This principle links the structure of universals to the structure of objects - universals have constituents when the objects that instantiate them have parts. The second principle provides an intuitively plausible connection between the constituency relation and the resemblance relation. Let " $x<y$ " mean " $y$ has all of the constituents of $x$ but $x$ does not have all of the constituents of $y$," where $x$ and $y$ are universals. Call this the resemblance principle: $a$ is more similar to $b$ than to $c$, and $c$ is more similar to $b$ than to $a$, iff $a<b<c$. This principle links resemblance to constituency - two properties are similar when they share constituents. Note that the resemblance principle applies only in cases where $a, b$, and $c$ share at least one constituent. If they have no constituents in common, they are utterly dissimilar and so cannot be compared along any axis of similarity. ${ }^{8}$

The constituency principle does a tremendous amount of work for Armstrong. First, it provides an algorithm for determining the constituents of structural universals. It explains, for example, why a charge universal is never a constituent of a mass universal - because not every massive object has a charged proper part. It also explains why a mass universal never has constituents of greater

\footnotetext{
${ }^{8}$ For supporting texts, see especially Armstrong (1978, pg. 116-131), (1988,pg. 312-316), and (1989a, pg. 106-107).
} 
mass - because no object with mass $x$ ever has a proper part with a mass greater than $x$. Second, the constituency principle grounds the structure of quantitative universals in the structure of objects and their proper parts. In so doing, it provides Armstrong with a justification for constructing quantitative universals as he does. Without this principle, we must brutely posit what the constituents of quantitative universals are. Tying resemblance to constituents that are themselves ungrounded is not an improvement over positing primitive resemblance.

Thus, Armstrong analyzes resemblance in two steps. First, the constituency principle grounds the ontology of universals in the ontology of objects; second, the resemblance principle uses the ontology of universals to ground resemblance relations.

In the following sections, I present several problems with this analysis. I conclude that Armstrong's account is not a plausible theory of quantitative properties, and thus is not an improvement over accounts that posit primitive resemblance.

\section{The Metric Function}

Armstrong uses mass as a paradigm example to illustrate his account of quantities, so I will focus on it in the next two sections. However, my criticisms in these sections apply to any quantitative property. For now, I shall assume that mass is spread out over regions, i.e. that only occupants of regions with finite volume have mass. On Armstrong's picture, every mass universal has smaller mass universals as constituents. For instance, the five grams mass universal has the universals four grams mass, three grams mass, etc., as constituents.

Take three mass universals: two grams mass, three grams mass, and two thousand grams mass. According to the constituency principle, the following is true: two grams mass shares all of its constituents with three grams mass, and three grams mass shares all of its constituents with two thousand grams mass, but two thousand grams mass does not share all of its constituents with three grams mass, and three grams mass does not share all of its constituents with two grams mass. Apply the resemblance principle, and two thousand grams mass is more similar to three grams mass than to two grams mass, and two grams mass is more similar to three grams mass than to two thousand grams mass. This provides Armstrong with an intuitive resemblance ordering: two grams mass is "smaller" than three grams mass, which is "smaller" than two thousand grams mass - therefore two grams mass is more similar to three grams mass than to two thousand grams mass. 
But this structure alone does not entail the resemblance relations that Armstrong claims it does. ${ }^{9}$ So far, all we have is an ordering of the mass universals. Consider three mass universals, $a, b$, and $c$, such that $a<b<c$. This tells us that $a$ is closer to $b$ than to $c$ (and that $c$ is closer to $b$ than to $a$ ), but not whether $b$ is closer to $a$ or to $c$. We know the ordering of $a, b$, and $c$; what we do not know are the distances between these universals.

Although the resemblance principle provides nothing more than a resemblance ordering, there are natural ways to extend the principle to capture the distances between mass universals. Here is one way. Let " $x<_{d} y$ " mean " $y$ has all of the constituents of $x$ and $x$ does not have $d$ constituents of $y$ (where $d$ is a number)." Now we can apply the following principle: If $a<_{m} b<_{n} c$, then $b$ is more similar to $a$ than to $c$ if and only if $m$ is less than $n$. Applied to universals $a$, $b$, and $c$, this extension appears to tell us how many constituents "away" $b$ is from both $a$ and $c$, and thus which universal it resembles more.

Unfortunately, this resemblance principle does no better than the original. This is easy to see if we assume that the mass quantities are isomorphic to the real numbers; i.e. that there are an uncountably infinite number of mass quantities. Every mass universal has an infinite number of constituents, so any two mass universals will have an infinite number of constituents in common as well as an infinite number not in common. So this resemblance principle will not yield the result that three grams mass and two grams mass are more similar three grams mass and two thousand grams mass. ${ }^{10}$

What we need to know is how "far apart" the mass quantities are. We can do this by using a mathematical metric. A metric is a function $d(x, y)$ defined on a set that assigns a non-negative value to each pair of elements. For all $x, y$, and $z$ in the set, the metric satisfies the following properties: (1) the value it assigns to ( $x$,

\footnotetext{
${ }^{9}$ See Armstrong (1978,pg. 116-131).

${ }^{10}$ The same problem arises if there are countably infinite numbers of constituents. In fact, Armstrong's account cannot guarantee the correct resemblance relations even if universals had finite numbers of constituents. Suppose there were only three mass universals: $a, b$, and $c$, and that $a$ is simple, $a$ is the only constituent of $b$, and $a$ and $b$ are the only constituents of $c$. According to Armstrong's resemblance principle, $b$ is equally similar to $a$ as to $c$. Now suppose that $a$ is 1 gram, $b$ is 10 grams, and $c$ is 11 grams. It is clear that the resemblance principle gives us the $w$ rong answer: 10 grams is not equally similar to $11 \mathrm{grams}$ and to $1 \mathrm{gram}$. This shows us that constituency facts do not ground resemblance; rather, it is the facts about the number of grams (i.e. the facts about the metric structure of the property) that play that role. When constituency facts and metric facts come apart, Armstrong's account delivers the wrong similarity judgments. And even if, fortuitously, they do not come apart, Armstrong's account is fundamentally mistaken about which facts ultimately ground resemblance.
} 
$y$ ) equals the value it assigns to $(y, x),(2)$ it assigns 0 to $(x, y)$ if and only if $x=y$, and $(3) d(x, z) \leq d(x, y)+d(y, z) \cdot{ }^{11}$

Intuitively, a metric tells us how close any two values are to one another.

For instance, a mass metric function might tell us that the distance between two grams mass and ten grams mass is eight grams, and the distance between ten grams mass and twelve grams mass is two grams. The mass metric function captures both the ordering of mass quantities as well as the distances between them. Thus it gives us everything we need to account for resemblance among mass quantities: any two quantitative universals are similar to the extent to which the distance (given to us by the metric function) between them is minimal. So, ten grams mass is more similar to two grams mass than to one hundred grams mass because the distance between ten grams mass and two grams mass is less than the distance between ten grams mass and one hundred grams mass.

Let's step back and assess Armstrong's position. Armstrong's constituency relation provides an ordering of quantities, but not the distances between them. In order to capture these distances, Armstrong must en rich his account by adding structure that encodes metric information.*

So where does this leave Armstrong with respect to other accounts of quantity? The boon of a theory of universals, according to Armstrong, is its ability to capture resemblance facts without the extra structure required by rival theories. We've seen, however, that both he and his rivals must incorporate metric structure in order to capture resemblance facts. So far, Armstrong's account has no advantage over any competing accounts. ${ }^{12}$

\section{Point Particles}

Grant Armstrong a metric function over the mass quantities. His account runs into further problems when we abandon the assumption that mass is only instantiated by objects spread out over regions. Recall the constituency principle, which states that a universal $x$ is a constituent of $y$ iff every object that instantiates $y$ has a proper part that instantiates $x$. So no object without proper parts can instantiate a structural universal. Now take a single electron. An electron is

\footnotetext{
${ }^{11}$ See Abbott (2001,pg. 222).

* The published version of this paper says that the metric function encodes information about the ordering of quantitative universals. That is incorrect: the metric provides information about the distance structure of quantities, but it does not provide an ordering.

${ }^{12}$ One might follow Hartry Field (1980) in grounding metrical assignments on a fundamental level of relations of congruence and betweenness. But even then the relevant judgments of resemblance that Armstrong is interested in would be captured by the pattern of distribution of these relations and not by the facts of constituency.
} 
point-sized - it has no radius and no apparent proper parts - yet it has a finite mass. How can this be?

Armstrong suggests that point particles like electrons do have proper parts - an infinite number of them. At every spacetime point where an electron is located, Armstrong claims there are an infinite number of particles. ${ }^{13}$ Each of these particles is a proper part of the single electron, and each has a part of the electron's mass. The mass property instantiated by the point-sized particle now satisfies the constituency principle, and it appears the problem is solved.

But this solution is unsatisfactory for several reasons. First, on this account it is not metaphysically possible that just one object which instantiates a quantitative property like mass can occupy a point at a time. This is highly counterintuitive. Not only is it possible that one object could occupy a spacetime region, it seems very plausible that this is actually the case.

Second, there is an important disanalogy between Armstrong's treatment of quantitative properties instantiated by point particles and those instantiated by objects spread out over a region. Armstrong claims that any point particle instantiating a quantitative property has the same pattern of parts that it would have if it were spread out. If this is true, it seems we should be able to isolate the parts of point particles just as we can isolate the parts of spread out objects. But it seems we cannot. For example, on Armstrong's account particles with half of the mass of the electron must exist, but to date physics has found no evidence of such particles. Why is this? Is it because there are heretofore unknown laws of physics preventing them from existing independently? The burden is on Armstrong to explain why these particles elude us.

Finally, we have no independent justification for Armstrong's assumption that every point particle has an uncountably infinite number of parts. Recall that Armstrong grounds the construction of universals in the mereology of objects. This tactic seems plausible in cases where we already have good reason to believe in these mereological relations, prior to the adoption of some particular metaphysical theory of properties. So, for example, it is plausible to postulate proper parts of a massy object when that object is extended over a region. This is not the case for point particles. The only reason we have for assuming the parts of point particles exist is that Armstrong's account requires their existence. Similar concerns apply, mutatis mutandis, to cases in which spacetime points instantiate fundamental field values. Just as Armstrong required point particles to have an infinite number of further point particles as parts, here he requires every spacetime point to have an

\footnotetext{
${ }^{13}$ Armstrong (1988, pg. 315).
} 
infinite number of further spacetime points as parts. A theory that posits infinitely many spacetime points at each spacetime location just to salvage a rather abstruse principle about universals is the sort of theory that many philosophers will find unappealing.

\section{Positive and Negative Quantities}

Armstrong seems to assume that his theory generalizes from the paradigm of mass to all fundamental quantitative properties. But this isn't the case. In this section, I will look at the fundamental property of charge. As with mass, Armstrong needs a metric function to capture resemblance between charge properties. And, as with mass, problems arise when we consider finite charge values instantiated at points. So let's grant Armstrong a similarity metric over charge properties, and let's assume that charge is only instantiated by objects spread out over regions of spacetime.

Although Armstrong provides a general sketch for the construction of quantitative universals, he never explicitly discusses charge. It is safe to assume, though, that Armstrong believes charge universals are constructed in the same way as any other fundamental quantitative property. Charge properties - like mass are structural universals whose constituents are other charge universals of smaller magnitudes. For example, a charge universal of +2 coulombs has constituents with values between 2 and 0 coulombs, and a charge universal of -2 coulombs has constituents with values between -2 and 0 coulombs.

An immediate problem emerges with this construction: positive and negative charge properties share no constituent universals. By the resemblance principle, positive and negative charges do not resemble each other at all; they have as much in common with one another as each has with mass. But surely this isn't right. Positive charge has something very important in common with negative charge - they are both charge!

How can Armstrong account for the apparent resemblance between positive and negative charge? Here is one option. Even though positive and negative charge do not share constituent universals, perhaps their similarity lies in the role they play in the laws of nature. So any resemblance between positive and negative charge consists solely in how they are treated by the laws. But this is resemblance in virtue of causal role, not in virtue of shared universals. Such a solution undercuts the motivation behind Armstrong's theory of structural universals, since the point of the project is to reduce resemblance to partial identity. 
Here is another option. Suppose we assume that properties of positive and negative charge do share constituent universals. In that case, every charge universal has constituents in common with every other charge universal - both positive and negative. Resemblance between positive and negative charge naturally follows.

While this option is attractive in some regards, it fails on several counts. First, it leads to odd constraints on metaphysical possibility. Recall that the constituency principle states an object instantiating a universal must have parts instantiating the constituents of the universal. On this version of Armstrong's account, then, any object that instantiates positive (or negative) charge must have positively and negatively charged parts. This rules out common cases in classical mechanics according to which there are such things as spheres of uniform positive charge. On this account such a sphere must have a part that instantiates negative charge - but as described, the sphere has no parts that instantiate negative charge. It turns out that many common classical mechanical cases are metaphysically impossible.

Second, problems arise concerning the construction of charge universals. What constituents does, say, the +3 coulombs universal have? Following Armstrong's construction of mass universals, it is natural to take charge universals to have constituents of smaller charge magnitudes. So +3 coulombs has a constituent for every charge value between +3 and $-3 .{ }^{14}$

But now we cannot deliver resemblance relations via the resemblance principle. Charge is an additive property: if we partition a charged object (divide it in to mutually exclusive and exhaustive parts) the charge of these parts will add up to the charge of the whole. Positive and negative values will cancel each other out, so that an object with a -10 coulombs part and $a+10$ coulombs part has zero net charge.

Take an object of +10 coulombs. We've assumed that such an object has a part for every charge value between +10 and -10 coulombs. So let's pick out a part of the object that has -9 coulombs of charge; call this part $a$ and the remainder part $b$. In order to preserve the charge value of the whole ( +10 coulombs), part $b$ must have +19 coulombs of charge. Now, we know that $b$ likewise has a part with every charge value between +19 and -19 coulombs. So let's pick out a part of $b$ that has -18 coulombs of charge; call this part $c$ and the remainder part $d$. Again, in order to preserve the charge value, the part $d$ must have a charge of +37 coulombs. And

\footnotetext{
${ }^{14}$ This is a natural move, I believe, but notice that we must simply assume that charged objects always have these parts. Already the theory is beginning to look uncomfortably ad hoc.
} 
so on. Eventually, we find that every charged object has proper parts that instantiate arbitrarily large and arbitrarily small positive and negative charge magnitudes. For any charge magnitude $c$, every charged object will have a part that instantiates $c$.

By the constituency principle, every charged object has parts that instantiate every possible charge value. So every charge universal will have constituents of every possible charge value. Thus, all charge universals have the same constituents, and so they all have the same constituents in common. But then, what grounds resemblance? Not the resemblance principle (in conjunction with the similarity metric), which tells us that the more constituents two universals share, the more they resemble each other. So Armstrong is still left without an account of resemblance.

(This account runs into further trouble if Armstrong takes two universals with the very same constituents to be numerically identical. By definition, qualitatively identical simple universals are numerically identical, but it is unclear how Armstrong deals with qualitatively identical structural universals. If universals with the same constituents are numerically identical, then every charge universal is numerically identical to every other charge universal - so a +2 coulomb universal would be identical to a +100 coulomb universal, an undesirable result.)

This extension of Armstrong's account fails. Perhaps other extensions will be contrived. But it's hard to see how any account will be able to capture all of the resemblance facts in a manner true to Armstrong's initial ambitions. In any case, I will not explore this further. One of the goals of a reductive project is to purge our ontology of dubious and otherwise unjustified entities. A reduction of resemblance that generates a dubious and otherwise unjustified ontology is not a reduction worth pursuing.

\section{Vector Quantities}

A vector is a quantity characterized by a magnitude and a direction. Any account of resemblance between properties needs to account for resemblance between vector properties.

Armstrong discusses vector properties in Armstrong (1988). He begins by allowing himself an ontology of fundamental properties and relations instantiated by spacetime points and intervals, but he does not allow any fundamental quantitative relations. He then claims that all vector properties can be reduced to these fundamental properties and relations. He backs up this claims with two 
examples: being $x$ amount later than and being $x$ amount east of. Both of these vector quantities may be reduced to a magnitude and spatiotemporal distance relation. ${ }^{15}$

But there are vector properties that cannot be reduced in this way. An electric field vector, for example, cannot be decomposed into electric field magnitudes and spatiotemporal interval properties. The pattern of electric field "magnitudes" and the spatiotemporal in tervals between them leaves out crucial information given by the electric field vector. In particular, it will not tell us what direction each magnitude "points" in. Consider a polarized ray of light - the locations and values of the ray's electric field magnitudes alone do not give the direction of polarization. So it is not the case that all vector properties can be reduced in the way Armstrong suggests.

Armstrong must enrich his fundamental ontology in some way if he wishes to account for vector properties. Here are three natural ways Armstrong might do this. First, he might be more liberal in the fundamental relations that he allows into his ontology. Second, he might decompose vectors into two fundamental properties. Third, he might simply allow fundamental vector properties into his ontology. Each of these strategies has its pitfalls, but the one they all share is a failure to account for resemblance.

Let's see how the first strategy of allowing fundamental relations works for electric field vectors. One way to pursue this strategy is to add two new fundamental elements to one's ontology: an electric field magnitude property and an electric field points-at relation. The electric field magnitude is the value associated with the electric field vector, and the electric field points-at relation is an asymmetric relation that holds between pairs of spacetime points that encodes the vector's direction. A 5 volts/meter electric field vector pointing north is reduced to a $5 \mathrm{v} / \mathrm{m}$ electric field magnitude and the fact that the electric field points-at relation holds between the spacetime point instantiating that magnitude and each spacetime point due north of it.

This version is unsatisfactory for several reasons. One of Armstrong's goals in providing a reductive account of vectors is to reduce the number of strange and unnecessary primitives in his ontology. But this version does the opposite - it increases both the number and the types of primitives required, and these primitives are arguably more exotic than the vector properties being reduced. Moreover, this account results in bizarre metaphysical possibilities. Armstrong believes in a combinatorial theory of possibility, according to which the

\footnotetext{
${ }^{15}$ Armstrong (1988,pg. 310-311).
} 
fundamental properties and relations of our world can be recombined to produce a possible world. ${ }^{16}$ If electric field magnitudes and electric field points-at relations are fundamental, then it is metaphysically possible that one could be instantiated without the other. In other words, two points could stand in the electric field points-at relation even though an electric field magnitude is not associated with either point.

A second way Armstrong might try to analyze vectors is by invoking pairs of monadic properties. For example, he might postulate a magnitude property that encodes the magnitude of the vector, and a direction property that encodes its direction. There are an infinite number of direction properties, one for each direction in which a vector can point. This strategy has the same demerits as the last one. It increases the number and types of primitives in Armstrong's ontology, and his combinatorialism entails that an electric field direction property may be instantiated at a point with no associated electric field magnitude, a strange consequence.

More importantly, neither of these accounts can fully capture resemblance between vector properties. Different directions resemble each other to different degrees. Consider three electric field vectors, $a, b$, and $c$, of the same magnitude, with the same origin, and lying in the same plane. Vector $a$ points north, $b$ points $10^{\circ}$ in a clockwise direction from $a$, and $c$ points $10^{\circ}$ clockwise from $b$. It seems that $a$ is more similar to $b$ than to $c$ because $a$ and $b$ are more similar with regard to direction.

Any account of resemblance must explain this, but an analysis of vectors in terms of either magnitudes and direction properties or magnitudes and points-at relations cannot. On Armstrong's account only quantitative fundamental universals have constituents, and neither direction properties nor points-at relations are quantitative. No direction universals have any constituents in common, so no direction universal ever resembles another direction universal. Likewise, no points-at relations have any constituents in common, so no points-at relation ever resembles another points-at relation. Thus, we cannot say that vector $a$ resembles $b$ more than $c$ because $a$ and $b$ point in more similar directions.

Neither strategy can capture all of the resemblance relations between vectors, since the sharing of constituents cannot explain any similarity between directions.

A third way Armstrong might try to account for vectors is by simply admitting them as primitives into his ontology. Yet problems arise here as well. Suppose every vector universal has constituents. Since vectors are instantiated at

\footnotetext{
${ }^{16}$ See Armstrong (1989b).
} 
points, this leads to the kinds of problems discussed in section 3, those that arise when finite magnitudes are instantiated at points. But unlike mass and charge, there is no story to tell about vector properties according to which they are extensive or spread out over a region. So in this case we do not even have a spread-out template that we can use to discern the structure of vector universals.

Resemblance is even more of a problem on this account than on the previous two. By the resemblance principle, two vector universals resemble each other when they have constituents in common. Suppose that a vector's constituents are vectors with smaller magnitudes that all point in the same direction. Then Armstrong can account for the similarity of vector universals that point in the same direction.

But he cannot account for the similarity of vector universals that point in different directions. (Any attempt to construct a vector property with constituents that point in different directions will run into the same troubles as we saw when we tried to construct charge properties with positive and negative constituents (see section 4).) Consider two unit electric field vectors $10^{\circ}$ apart that have the same origin and lie in the same plane. On this account, they do not resemble each at all, since they share no constituents. But it seems they have a great deal in common; in fact, they exactly resemble in several respects. First, they are both vectors, and two vector properties are more similar than a vector and a scalar property. Second, they both electric field vectors, and two electric field vector are more similar than an electric field vector and a magnetic field vector. Third, they have the same magnitude, and two vectors of the same magnitude pointing in different directions are more similar than two vectors of different magnitudes pointing in different directions. Yet on this account these two electric field vectors resemble each other no more than each resembles a mass universal.

I shall not speculate about how Armstrong would revise his theory of vectors when faced with the decisive problems with the account he actually states. I have no idea which package of costs would seem more palatable and, in particular, to what extent he would be willing to accept additional fundamental relations, to what extent he would be willing to deny the authenticity of various apparent resemblance facts, and to what extent he would be willing to bite various bullets on combinatorial possibility. What is inevitable is a breakdown of the original picture that sought to explain all intrinsic resemblance by the sharing of constituents. At best, Armstrong cannot capture all the resemblances between vector properties, and at worst he cannot accommodate vector properties at all. 


\section{Resemblance in Different Respects}

It is no surprise that our resemblance judgments depend significantly on context. Armstrong lauds universals for readily explaining this phenomenon when it comes to comparing similar objects: "If resemblance is a matter of different identities in different cases, it is easy to see that degrees of resemblance will be a partially subjective matter, depending upon what particular properties we happen to be interested in, in the particular context." ${ }^{17}$ But while Armstrong can accommodate context-sensitivity when it comes to objects, he cannot do the same for properties.

Consider three charge universals: +5 coulombs, -1 coulomb, and -5 coulombs. One way to judge similarity between them is in terms of difference in charge value - the -5 coulombs universal is more similar to the -1 coulomb universal than it is to the +5 coulombs universal because -1 and -5 are only four units of charge "apart", while -5 and +5 are ten units "apart". But there is another way to judge similarity. We could say instead that the -5 coulombs universal is more similar to the +5 coulombs universal than the -1 coulomb universal because 5 and +5 are the same magnitude of charge. Neither judgment of resemblance is prohibited; rather, the first seems right when similarity is judged in one context, the second seems right when similarity is judged in another.

The same can be said for vector quantities. Consider three unit electric field vectors lying on the same plane with a common origin: one points north, one points west, and one points south. Again, which are more similar? On the one hand, the vector pointing north is more similar to the one pointing west because the angle between them is smaller. On the other, the vector pointing north and the vector pointing south are more similar because they are parallel, while the vector pointing west is perpendicular.

This generates a challenge for Armstrong. We have seen how he wishes to account for the context-dependence of resemblance judgments in the case of objects - we restrict our attention to a subset of the universals in play. And we have seen that there is certainly context dependence of resemblance judgment in the case of magnitudes. For his account of resemblance to have desirable unity, he would need to explain context dependence in the same way: in terms of attention being restricted to a subset of the universals in play. But in the cases described above, there seem to be more dimensions of context-dependence to judgments of resemblance than there are families of universals present. Some kind of breakdown in the account once again seems imminent.

\footnotetext{
${ }^{17}$ Armstrong (1988,pg. 103).
} 


\section{Conclusion}

Why universals?

For Armstrong, a major selling point of his theory of properties is its power to analyze resemblance. Universals can account for our resemblance intuitions while providing a reduction of resemblance, he says. No other theory of properties can do the same.

Armstrong offers a theory of universals that is intended to provide a unified account of resemblance for both objects and properties. Objects instantiate universals, and the more universals objects share the more similar they are. Universals are constructed out of constituents, and the more constituents universals share the more similar they are. The construction of universals is linked to the construction of objects: only objects with the appropriate proper parts can instantiate structural universals.

We've seen that this construction of structural universals does not deliver an adequate account of resemblance among properties. First, appeal to the "number of" shared constituents is no substitute for a similarity metric. Second, the applications to point-sized particles are bizarre and ad hoc. Third, the account faces special troubles from positive and negative quantities. Fourth, it faces worse troubles from vector quantities. Finally, the theory provides no basis for a unified account of context-sensitivity for resemblance. Armstrong writes: "The fate of the Universals theory may turn on the question of the in exact resemblance of universals." 18

I concur. ${ }^{19}$

\section{References}

Stephen Abbott (2001). Understanding Analysis. New York: Springer-Verlag Press. D. M. Armstrong (1978). Universals and Scientific Realism, Volume II: A Theory of Universals. Cambridge University Press.

${ }^{18}$ Armstrong (1988,pg. 139).

${ }^{19}$ Many thanks to Frank Arntzenius, John Hawthorne, Ted Sider, and especially Chris Meacham for extensive comments. 
D. M. Armstrong (1983). What is a Law of Nature? New York: Cambridge University Press.

D. M. Armstrong (1988). "Are Quantities Relations? A Reply to Bigelow and Pargetter.” Philosophical Studies 54, pg. 305-316.

D. M. Armstrong (1989a). Universals: An Opinionated Introduction. Colorado: Westview Press.

D. M. Armstrong (1989b). A Combinatorial Theory of Possibility. New York: Cambridge University Press.

Hartry Field (1980). Science Without Numbers. Princeton: Princeton University Press.

David Lewis (1986). “Against Structural Universals.” Australasian Journal of Philosophy 64, pg. 25-46. Reprinted in Lewis, Papers in Metaphysics and Epistemology (1999), pg. 78-107. 